\title{
Article
}

\section{Detection of Spoofing Used Against the GNSS-Like Underwater Navigation Systems}

\author{
Tomasz Abramowski ${ }^{1}$, Mateusz Bilewski ${ }^{2}$, Larisa Dobryakova ${ }^{3}$, Evgeny Ochin ${ }^{4,}$, Janusz Uriasz \\ ${ }^{5}$ and Paweł Zalewski ${ }^{6}$ \\ The names of the authors are sorted alphabetically: \\ 1 Maritime University of Szczecin, Faculty of Navigation, Szczecin, Poland; t.abramowski@am.szczecin.pl, \\ ORCID iD https://orcid.org/0000-0002-9029-406X \\ 2 Maritime University of Szczecin, Faculty of Navigation, Szczecin, Poland; m.bilewski@am.szczecin.pl, \\ ORCID iD https://orcid.org/0000-0003-1262-1309 \\ 3 West Pomeranian University of Technology, Faculty of Computer Science and Information Technologies, \\ Szczecin, Poland; ldobryakova@wi.zut.edu.pl, ORCID iD https://orcid.org/0000-0003-4433-3774 \\ 4 Maritime University of Szczecin, Faculty of Navigation, Szczecin, Poland; e.ochin@am.szczecin.pl, \\ ORCID iD https://orcid.org/0000-0003-4450-9151 \\ 5 Maritime University of Szczecin, Faculty of Navigation, Szczecin, Poland; j.uriasz@am.szczecin.pl \\ 6 Maritime University of Szczecin, Faculty of Navigation, Szczecin, Poland; p.zalewski@am.szczecin.pl, \\ ORCID iD https://orcid.org/0000-0001-8157-9728 \\ * Correspondence: e.ochin@am.szczecin.pl; Tel.: +48 608437562
}

\begin{abstract}
The purpose of the work is an underwater positioning safety study that used the GNSSlike underwater navigation systems. In the process of research, we used the methods of software modeling of underwater spoofing processes. The spoofing problem consists of three stages: design of spoofers, design of spoofing detection systems, and design of anti-spoofing systems. This article discusses some methods of spoofing detection. We briefly describe the known methods of underwater positioning systems. Unlike GNSS, currently only LNSS (Local Navigation Satellite System) can be considered in this case. Spoofing detection systems with one hydrophone are of great practical importance, as they allow for use of standard hydroacoustic equipment. However, detection of spoofing is not possible in static mode, which is with underwater vehicle at rest. In case of two hydrophones the detection of spoofing in static mode is possible. We discuss the navigation based on the use of an acoustically passive receiver. The receiver "listens" to the buoys and solves the problem of finding its own position using the coordinates of the buoys (such systems are called GNSS-like Underwater Positioning Systems or GNSS-like UPS). Depending on the scale of system service area, GNSS-like UPS-es are divided into global, regional, zonal and local systems. In this article, we take into account only the local class of GNSS-like UPS. The acoustic signal generator transmits a simulation of several buoy signals. If the level of the simulated signal exceeds the signal strength of actual buoys, the UPS receiver will "lock onto" the fake signal and then calculate a false position basing on it. The development of further research should be focused on the creation of hardware and software systems for conducting physical experiments at depths up to $400 \mathrm{~m}$.
\end{abstract}

Keywords: antiterrorism; underwater GNSS; underwater GPS; spoofer; antispoofing; spoofing detection; underwater transport safety

\section{Introduction}

In 2002 was led the adoption of Chapter XI-2 of the SOLAS-74 Convention and the International Ship and Port Facility Security Code (ISPS Code). In the 20th century, electronic devices such as radar and Loran were widely adopted for use in navigation. Today most vessels use an automatic pilot, an electronic device allowing vehicle control without constant human intervention. The use of GNSS (GPS Navstar, GLONASS, BeiDou, GALILEO, QZSS and NavIC) has become standard in navigation. 
The proven effectiveness of GNSS spoofing attacks on critical systems like maritime navigation equipment is increasingly recognized by US and other governments. A 2012 National Risk Estimate conducted by the US Department of Homeland Security found that "US critical infrastructure sectors are increasingly at risk from a growing dependency on the GPS for space-based position, navigation, and timing (PNT). GNSS spoofing attack scenarios in particular presented the highest consequences to critical national infrastructure due to the potential lapse of time between when the interference begins and when it is detected (Fig. 1) [1]

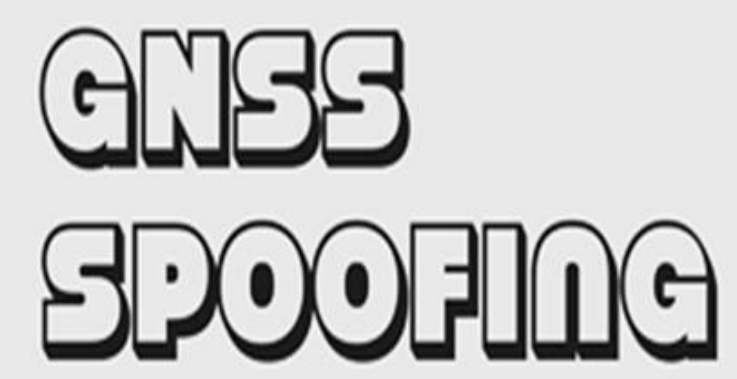

\section{Spoofing mimics authentic GNSS satellites to hijack GNSS receiver tracking loops.}
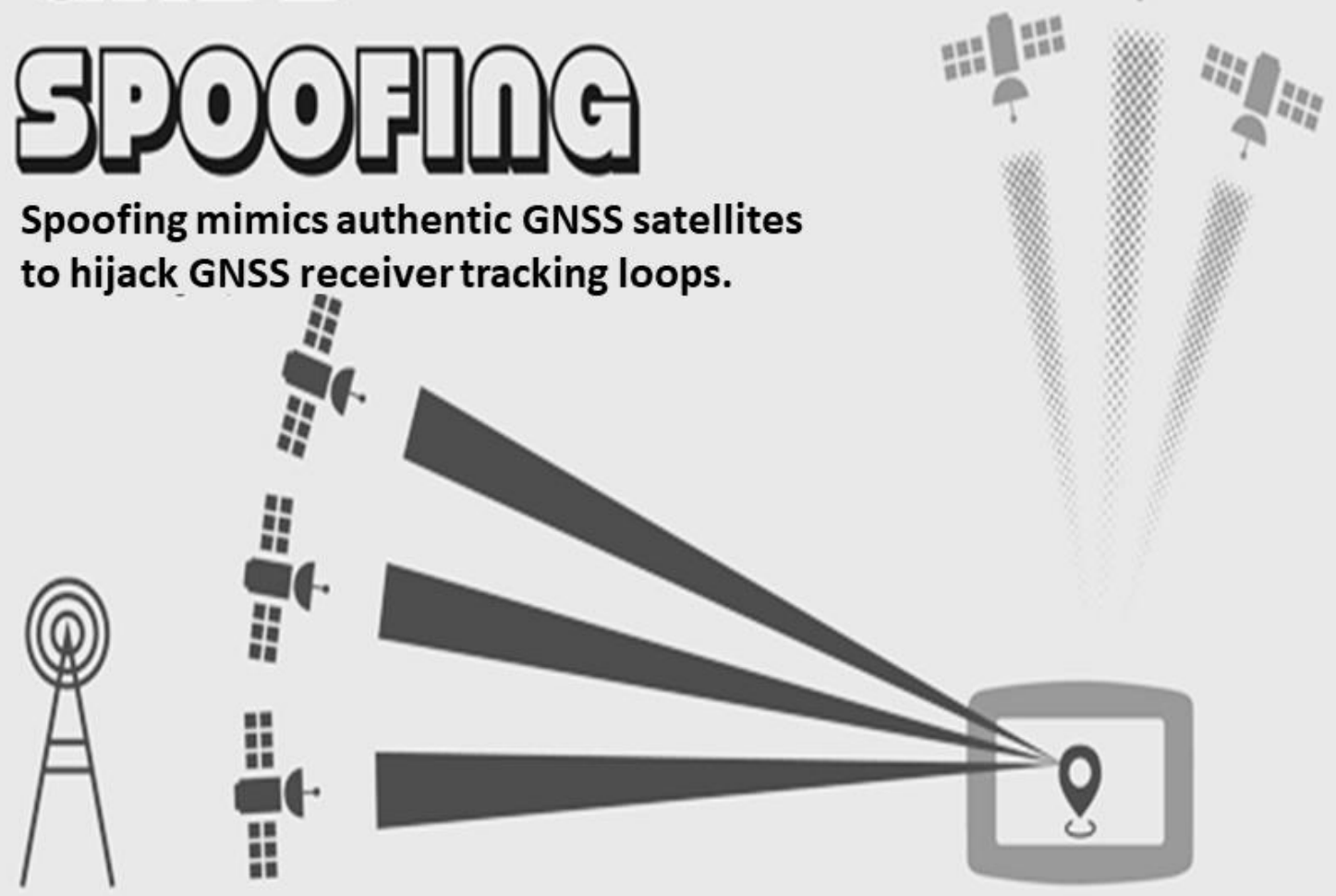

\section{Figure 1. GNSS Spoofing [1].}

GNSS is embedded in a wide range of basic day-to-day economic and transport functions, but many civilian systems remain vulnerable. Attackers could use multiple techniques to disrupt and even profit off these potential vulnerabilities. The main GNSS spoofing scenarios:

- Divert vessel into hostile or territorial waters

- Disrupt port activities by targeting cranes using GNSS for automated container logistics

- Hijack cargo in transit by disguising true location of container

If the problems of GNSS Spoofing and, accordingly, GNSS Anti-Spoofing are currently developed in sufficient detail, the problems of GNSS Underwater Spoofing are still in the state of initial research. Guided by sonar beacons located on the ocean bed, the robots will be able to accurately determine their own location down to millimeters and exchange data in real time with control stations, which may be air-, water- and ground-based. Buoys have three modes of operation. In the first one, a buoy receives information via satellite communication channels, records it and, at the request of the robot, transmits it. In the second mode - so called "dialogue" - the 
buoy connects the coastal, aerial, and marine control centers with underwater robots over the VHF radio channel in real-time mode. Such data exchange allows not only for knowing where the robot is and what tasks it solves, but also for exerting continuous control over it. The third mode is the simplest. The robot operates completely autonomously and only checks its coordinates with buoys, adjusting the course. In an emergency, the drone can emit an SOS signal, thus reporting the termination of its deep-sea mission.

Note the four main methods used in determining positions underwater, which largely coincide with the methods of measuring the coordinates of mobile objects in electromagnetic signal networks.

1. Received Signal Strength (RSS) - distance to the object is estimated by the power of the signal. This method works well at short distances.

2. Angle of Arrival (AoA) - the location of the object is determined within the area of a triangle formed by the intersection of the axes of the antenna patterns of the sectors of three base stations (modified trilateration method).

3. Round Trip Time (RTT) - the object sends a signal to the transceiver and waits for a response. The half-difference between the time of sending a signal by an object and receiving a signal by an object multiplied by the speed of light gives the distance between the transceiver and the object.

4. Time of Arrival (ToA) is a technique in which the time of arrival of a specific signal with precisely synchronized time of its sending, is calculated (this method requires time synchronization at the sender and the recipient).

\section{Literature Survey}

There are many manufacturers of underwater positioning systems in the world including iXblue [2], EvoLogics [3], Sonardyne [4] and Charles Stark Draper Laboratory [5]. A so-called Positioning System for Deep Ocean Navigation (POSYDON) program [6] aims to develop an undersea system, which would provide omnipresent, robust positioning across ocean basins. The development of this technology is outlined in the works [7-10].

In this article, we briefly describe the known methods of underwater positioning systems. Unlike GNSS, currently only a so-called LNSS (Local Navigation Satellite Systems) or GNSS-like, can be taken into account. Spoofing detection systems with one hydrophone are of great practical importance, as in this case it is possible to use standard hydro acoustic equipment. However, in static mode that is with underwater vehicle at rest the detection of spoofing is not possible. When two hydrophones are used, the detection of spoofing in static mode becomes possible.

The Spoofing-Antispoofing problem consists of three partial problems: design of spoofers, design of a spoofing detection system, and design of anti-spoofing systems. This article discusses some methods, which may be used for the detection of spoofing.

\section{Notations and Definitions}

UAV - Underwater Autonomous Vehicle. $z_{0}(x, y)-$ known depth of UAV (m).

$\boldsymbol{B}_{i} \rightarrow\left\{x_{i}, y_{i}, z_{i}\right\}, i=\overline{\mathbf{1}, \boldsymbol{N}}$ - buoys of GNSS-like UPS.

$\left\{\boldsymbol{x}_{v}, \boldsymbol{y}_{v}, z_{v}\right\}$ - coordinates measured by UAV(m).

$\left\{\tilde{x}_{v}, \tilde{y}_{v}, \tilde{z}_{v}\right\}-$ coordinates measured by the spoofer (m).

$\left\{\Delta x_{v}, \Delta y_{v}, \Delta z_{v}\right\}-$ correction of UAV coordinates (m). 
$\boldsymbol{T}_{\boldsymbol{i}}=\left(\boldsymbol{t}_{\boldsymbol{i}}^{\text {arrival }}-\boldsymbol{t}_{\boldsymbol{i}}^{\text {sent }}\right)-$ the measured signal's propagation time from the buoy $B_{i}$ to the spoofer (s).

GNSS Spoofing - substitution of satellite navigation data in order to deceive the target. Initially, the spoofer sends the correct coordinates, but then gradually shifts the signal to the side. Doing this slowly is necessary so that the GNSS receiver does not reject all signals due to an abrupt change in location.

Underwater GNSS-like or Underwater Local Navigation Satellite System (LNSS) - Underwater Positioning System (UPS) based on GNSS.

Underwater GNSS-like or Underwater LNSS Spoofing - substitution of navigation data emitted by surface radio-acoustic or underwater acoustic buoys in order to deceive the target. The spoofer can be a surface or underwater manned or unmanned vehicle.

\section{The Options Available for Building a GNSS-like Underwater Positioning Systems (UPS)}

\section{a. Wireless Buoyant GNSS-like UPS}

The wireless (acoustic) buoyant UPS (Fig. 2) have positioning accuracy $\varepsilon$ witch determined by the distances between an UAV and the buoys:

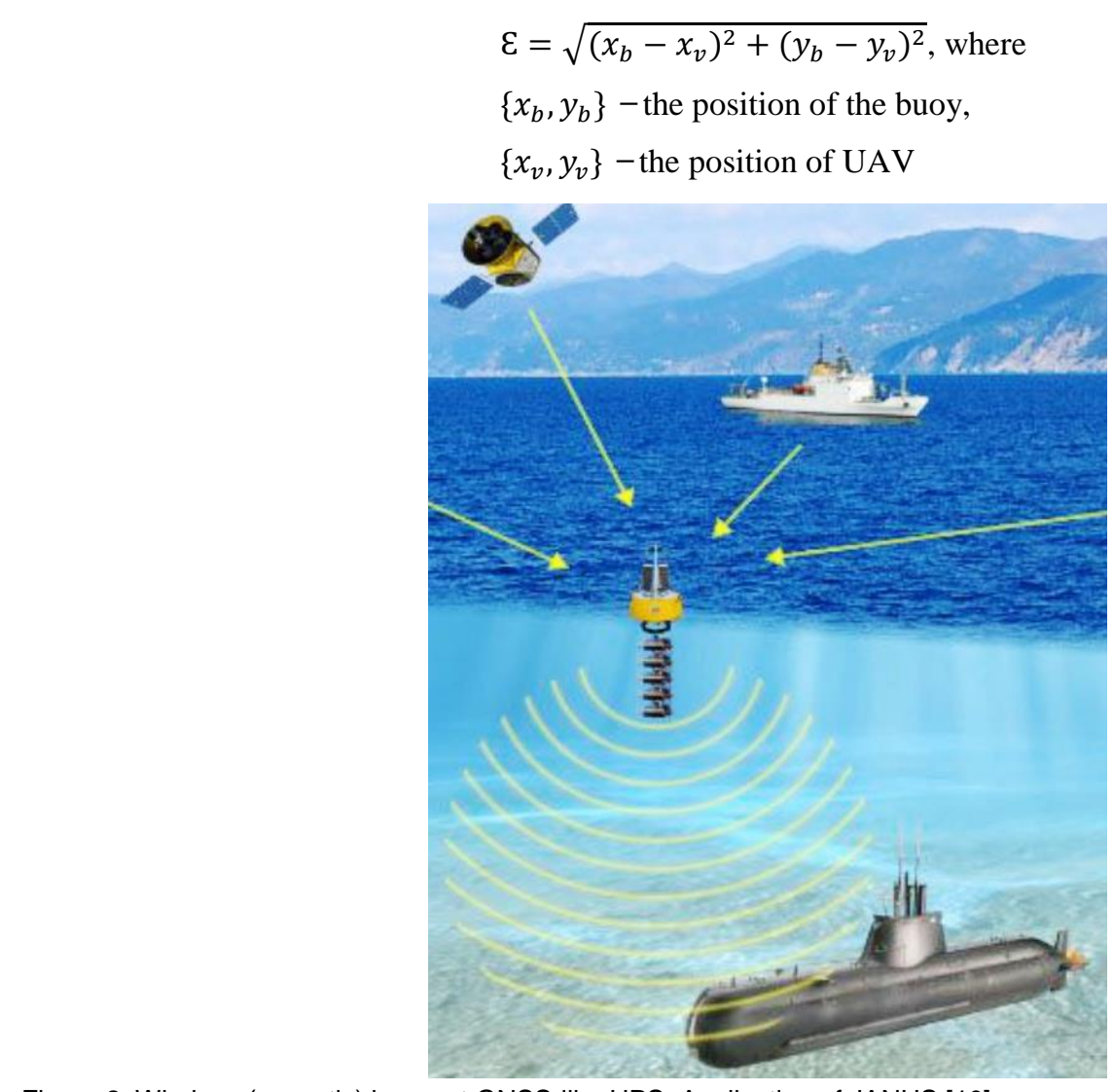

Figure 2. Wireless (acoustic) buoyant GNSS-like UPS. Application of JANUS [10].

\section{b. Direct GNSS-like UPS}

In 1992, Youngberg inspired a direct transposition of GNSS signal to underwater world [11-12] (Fig. 3). Acoustic waves directly go from surface buoys acting as satellites to the underwater receivers. 


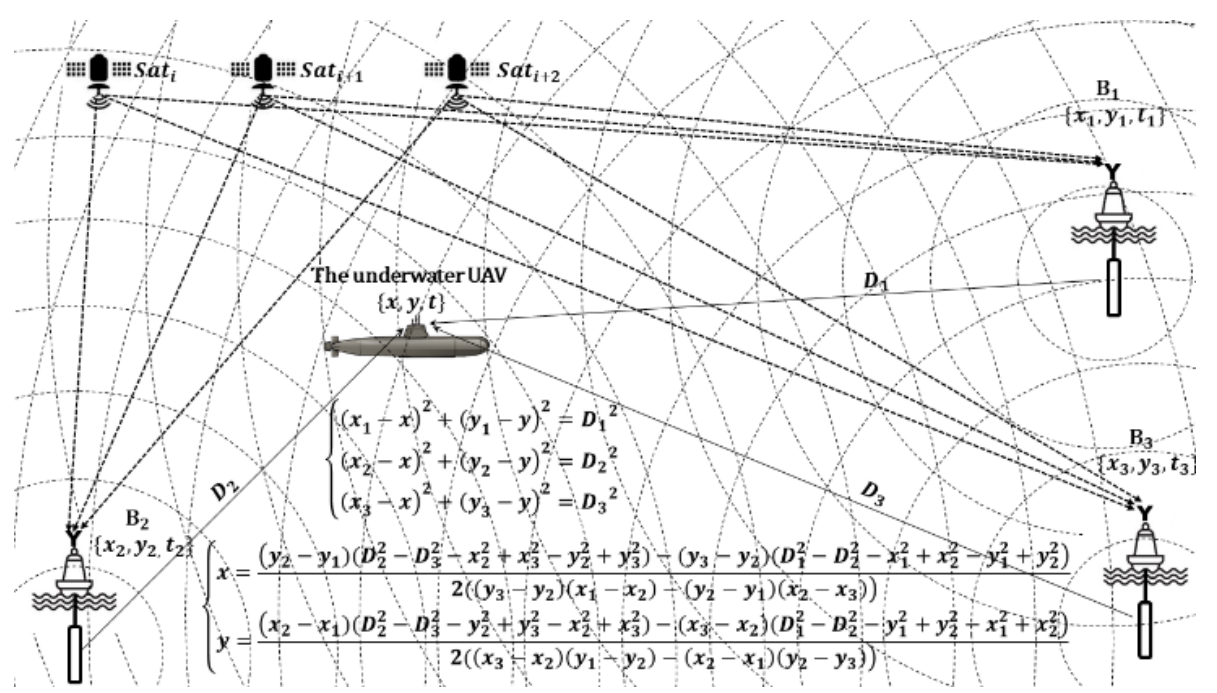

Figure 3. Direct GNSS-like UPS: $B_{1}, B_{2}$ and $B_{3}$ - sonar transponders of GNSS signals (2D case simulation result), UAV Underwater Autonomous Vehicle.

The surface buoys determine the $\mathrm{XY}$ coordinates $(\mathrm{Z}=0)$ and time $T$, based on which the receiver of GNSS-like signals determines the own XYZ coordinates. In some applications only the XY coordinates are significant, since the depth $\mathrm{Z}$ of the dive can be determined by a depth gauge, so we will focus only on the calculations of the XY coordinates.

In this case (2D) it can be shown without loss of generality (3D) that the system of equations (2)

$$
\left\{\begin{array}{l}
\left(x_{1}-x\right)^{2}+\left(y_{1}-y\right)^{2}=D_{1}^{2} \\
\left(x_{2}-x\right)^{2}+\left(y_{2}-y\right)^{2}=D_{2}^{2} \\
\left(x_{3}-x\right)^{2}+\left(y_{3}-y\right)^{2}=D_{3}^{2}
\end{array}\right.
$$

describing the relationship of buoy coordinates and UAV coordinates has the following solution (2):

$$
\left\{\begin{array}{l}
x=\frac{\left(y_{2}-y_{1}\right)\left(D_{2}^{2}-D_{3}^{2}-x_{2}^{2}+x_{3}^{2}-y_{2}^{2}+y_{3}^{2}\right)-\left(y_{3}-y_{2}\right)\left(D_{1}^{2}-D_{2}^{2}-x_{1}^{2}+x_{2}^{2}-y_{1}^{2}+y_{2}^{2}\right)}{2\left(\left(y_{3}-y_{2}\right)\left(x_{1}-x_{2}\right)-\left(y_{2}-y_{1}\right)\left(x_{2}-x_{3}\right)\right)} \\
y=\frac{\left(x_{2}-x_{1}\right)\left(D_{2}^{2}-D_{3}^{2}-y_{2}^{2}+y_{3}^{2}-x_{2}^{2}+x_{3}^{2}\right)-\left(x_{3}-x_{2}\right)\left(D_{1}^{2}-D_{2}^{2}-y_{1}^{2}+y_{2}^{2}-x_{1}^{2}+x_{2}^{2}\right)}{2\left(\left(x_{3}-x_{2}\right)\left(y_{1}-y_{2}\right)-\left(x_{2}-x_{1}\right)\left(y_{2}-y_{3}\right)\right)}
\end{array}\right.
$$

\section{The Main Strategy of Underwater GNSS-like Spoofing}

At the moment of target's capture false coordinates coincide with the real ones, and then simulate the movement of UAValong a certain trajectory.

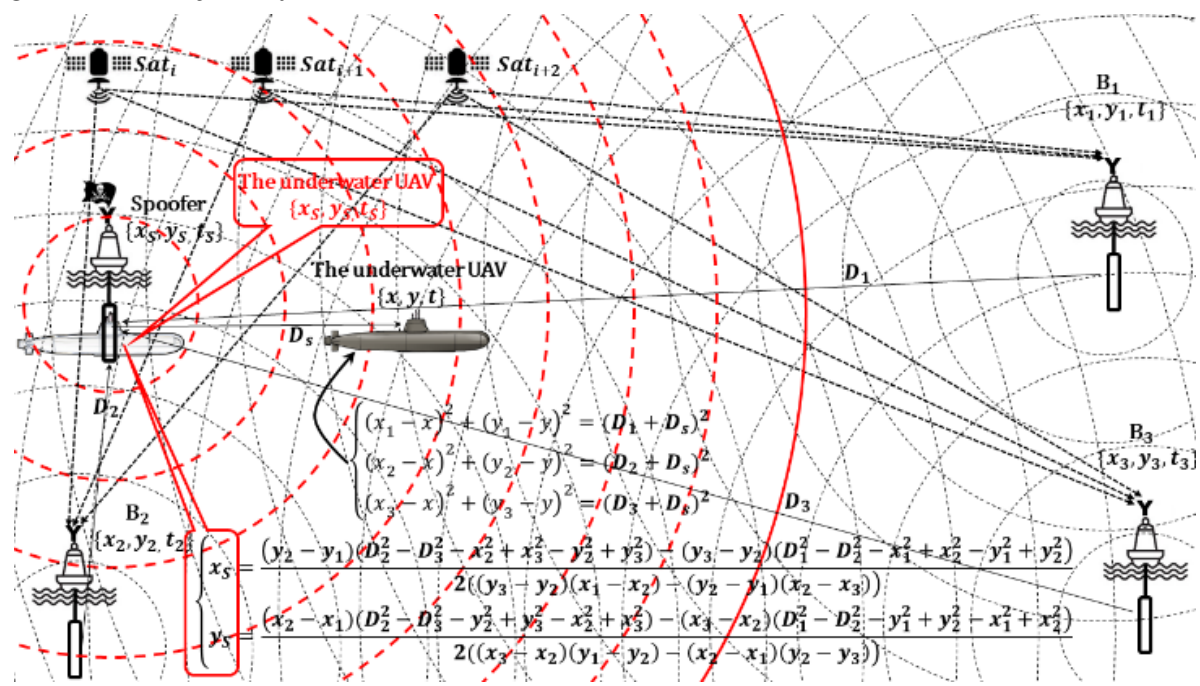


Figure 4. The main strategy of spoofing (2D case simulation result): D1, D2 and D3 - the distances from sonar transponders to spoofer; DS - the distance from the spoofer to UAV; $\left\{\mathrm{x}_{1}, \mathrm{y}_{1}, \mathrm{t}_{1}\right\},\left\{\mathrm{x}_{2}, \mathrm{y}_{2}, \mathrm{t}_{2}\right\}$ and $\left\{\mathrm{x}_{3}, \mathrm{y}_{3}, \mathrm{t}_{3}\right\}$ - the coordinates of sonar transponders and the exact time received from navigation satellites; the red continuous circle shows the boundary of the effect of spoofing.

As a spoofer, we use an acoustic signal repeater [13-14] (Fig. 4, highlighted in red). It can be shown that by solving the system of equations (4)

$$
\left\{\begin{array}{l}
\left(x_{1}-x_{S}\right)^{2}+\left(y_{1}-y_{S}\right)^{2}=D_{1}{ }^{2} \\
\left(x_{2}-x_{S}\right)^{2}+\left(y_{2}-y_{S}\right)^{2}=D_{2}{ }^{2} \\
\left(x_{3}-x_{S}\right)^{2}+\left(y_{3}-y_{S}\right)^{2}=D_{3}{ }^{2}
\end{array}\right.
$$

by analogy with (3), where the coordinates of the spoofer are $\left\{x_{S}, y_{S}\right\}$. In this case, the system of equations

$$
\left\{\begin{array}{l}
\left(x_{1}-x\right)^{2}+\left(y_{1}-y\right)^{2}=\left(D_{1}+D_{s}\right)^{2} \\
\left(x_{2}-x\right)^{2}+\left(y_{2}-y\right)^{2}=\left(D_{2}+D_{s}\right)^{2} \\
\left(x_{3}-x\right)^{2}+\left(y_{3}-y\right)^{2}=\left(D_{3}+D_{s}\right)^{2}
\end{array}\right.
$$

describing the relationship between the coordinates of buoys, the repeater of acoustic signals and the coordinates of the UAV has a single solution (4) under the condition $\boldsymbol{D}_{\boldsymbol{s}}=\mathbf{0}$ (Fig. 5), that is, all the UAVs that are in range of the spoofer (acoustic repeater) define their coordinates as $\left(x_{S}, \mathrm{y}_{S}\right)$.

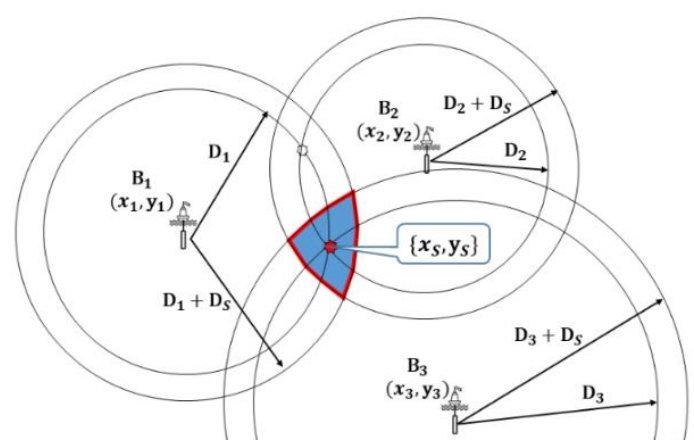

Figure 5. The relationship of the coordinates of the buoys, the spoofer (follower of acoustic signals) and the coordinates of the UAV has a unique solution (2) under the condition $\boldsymbol{D}_{\boldsymbol{s}}=\mathbf{0}$.

\section{The Examples of UAV Trajectory}

The motion's law of the mass center of a UAV in general may be represented by the system of two equations

$$
\left\{\begin{array}{l}
x=x(t) \\
y=y(t)
\end{array}\right.
$$

The UAV autopilot implements a discrete path calculation process (Fig. 7)

$$
\left\{\begin{array}{l}
x_{i+1}=x_{i}+\Delta x_{i} \\
y_{i+1}=y_{i}+\Delta y_{i}
\end{array}\right.
$$

where $\left\{x_{i}, y_{i}\right\}$ - current position of a mass center of the UAV; $\left\{\Delta x_{i}, \Delta y_{i}\right\}$ - estimated route correction values. 


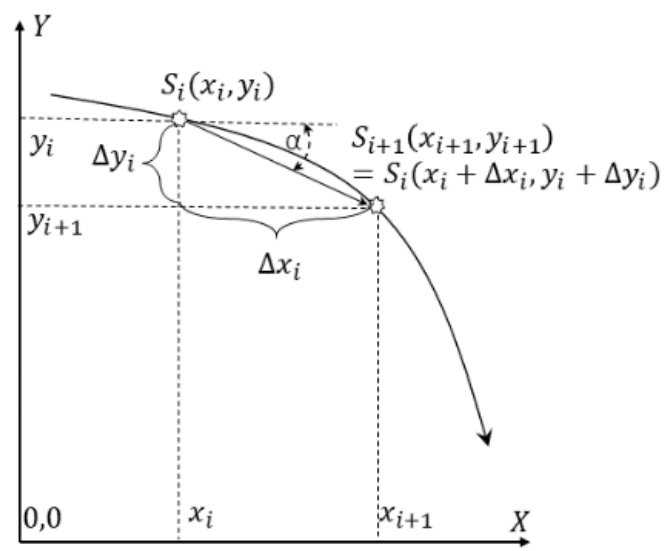

Figure 6. Estimated route correction values

To reach the point $\left\{x_{i+1}, y_{i+1}\right\}$ the UAV moves at an angle

$$
\alpha=\arctan \frac{y_{i+1}-y_{i}}{x_{i+1}-x_{i}},-\pi \leq \alpha \leq \pi
$$

Moving the mass center of the UAV from the position $\left\{x_{i}, y_{i}\right\}$ in position $\left\{x_{i+1}, y_{i+1}\right\}$ accompanied by random deviations from the route $\left\{\varepsilon x_{i}, \varepsilon y_{i}\right\}$, i.e.

$$
\left\{\begin{array}{l}
x_{i+1}:=x_{i+1}+\varepsilon x_{i} \\
y_{i+1}:=y_{i+1}+\varepsilon y_{i}
\end{array}\right.
$$

Suppose a UAV performs underwater circulation of a radius $R=1000 \mathrm{~m}$ with a speed $V_{U A V}=16 \mathrm{~km} / \mathrm{h}$, with time discretization $\Delta t=60 \mathrm{sec}$. In this case

$$
\left\{\begin{array}{l}
x_{i+1}=x_{i}+V_{U A V} \Delta t \cos \alpha+\aleph_{i} x_{i} / 100 \\
y_{i+1}=y_{i}+V_{U A V} \Delta t \sin \alpha+\aleph_{i} y_{i} / 100
\end{array}\right.
$$

where $\aleph_{i}-$ random number uniformly distributed in the interval $[0,1]$.

When the acoustic signal is low, the circulation is performed normally (Fig. 7). As the power of the acoustic signal increases, a truncated (limited) circulation is performed (Fig. 8).

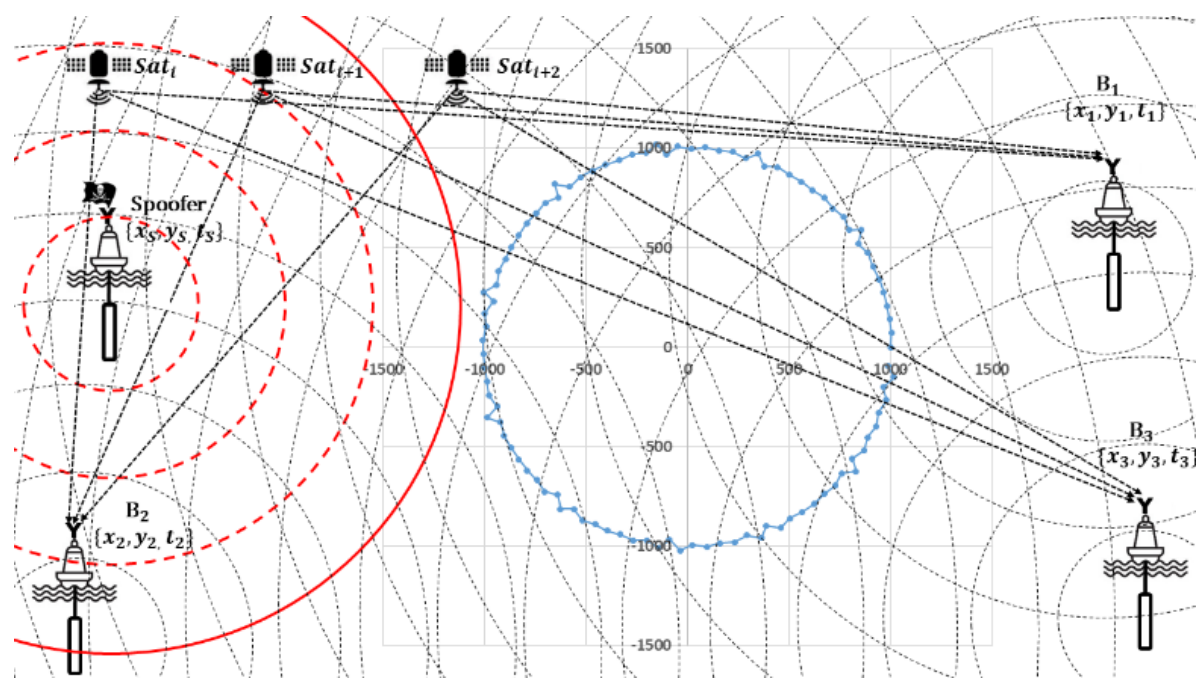

Figure 7. Normal circulation of UAV (2D case simulation result); $\left\{x_{s}, y_{s}\right\}=\{-3000 \mathrm{~m}, 200 \mathrm{~m}\}$; the red continuous circle with radius $1900 \mathrm{~m}$ shows the boundary of the effect of spoofing. On the UAV movement trajectory (marked in blue), we see the divergence of the one-step calculated and observational coordinates of the UAV in normal driving. The direction of the discrepancy is determined from the calculated UAV location to the observational coordinates. 


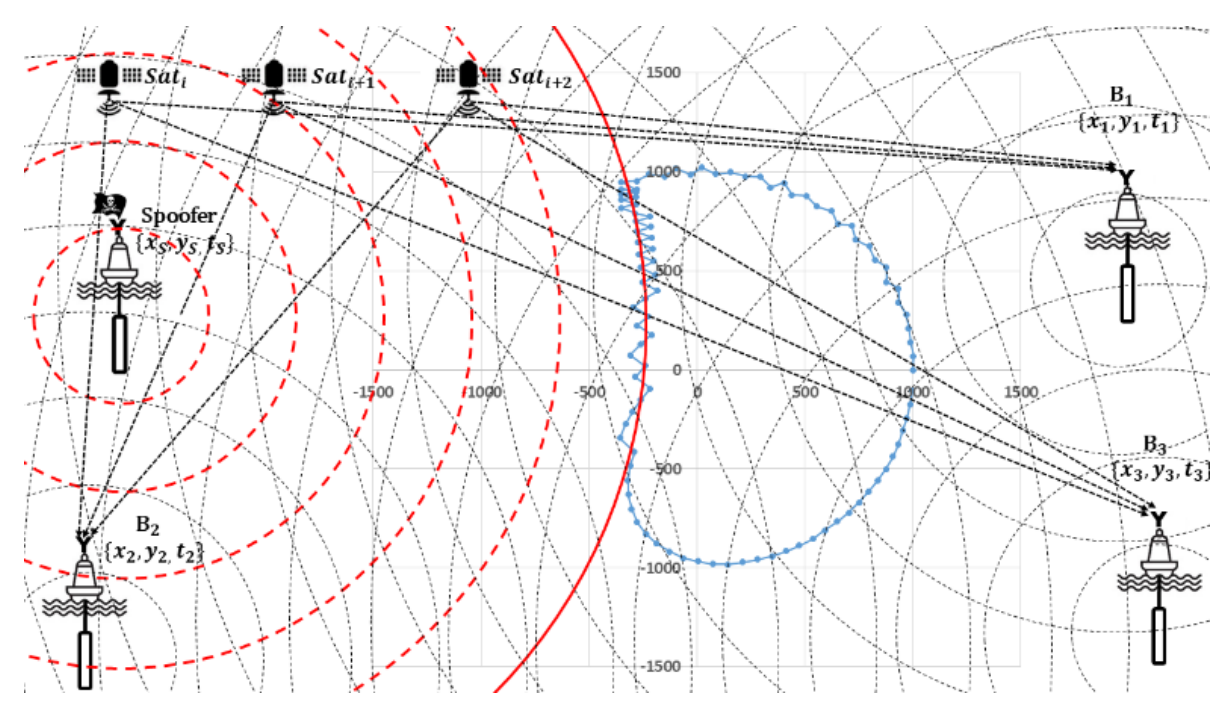

Figure 8. Truncated (restricted) circulation of an UAV as a result of spoofing (2D case simulation result); $\left\{\mathrm{x}_{\mathrm{s}}, \mathrm{y}_{\mathrm{s}}\right\}=\{-3000 \mathrm{~m}$, $200 \mathrm{~m}$; ; the red continuous circle with a radius of $2800 \mathrm{~m}$ shows the boundary of the effect of spoofing. On the UAV movement trajectory (marked in blue), we see the divergence of the one-step calculated and the observed coordinates of the UAV in the spoofing mode.

\section{The GNSS-like positioning of a spoofer and a target}

Solving the system of equations (2) allows us to calculate the target's coordinates

$$
\left\{x_{v}, y_{v}, z_{v}\right\}=\sqrt{\left(x_{i}-x_{v}\right)^{2}+\left(y_{i}-y_{v}\right)^{2}+\left(z_{i}-z_{v}\right)^{2}} \approx c T_{i}, \quad i=\overline{1, N}
$$

where $T_{i}$ - measured propagation time of a real signal from a buoy $B_{i}$ to the target.

The system of equations (11) is written as

$$
\varepsilon\left(x_{v}, y_{v}, z_{v}\right)=\sum_{i=1}^{N}\left(\sqrt{\left(x_{i}-x_{v}\right)^{2}+\left(y_{i}-y_{v}\right)^{2}+\left(z_{i}-z_{v}\right)^{2}}-c T_{i}\right)
$$

In general case, the solution of (12) is carried out by numerical minimization methods (13):

$$
\left\{x_{v}, y_{v}, z_{v}\right\}=\arg \min _{x_{v}, y_{v}, z_{v}} \varepsilon\left(x_{v}, y_{v}, z_{v}\right)
$$

There is enough data from three buoys to determine $\left\{x_{v}, y_{v}, z_{v}\right\}$, however, as the software simulation of GNSSlike UPS shows, due to the approximate nature of the measurement of pseudoranges $\left(\rho_{i} \approx c T_{i}, i=1, N\right)$ the positioning accuracy $\left\{x_{v}, y_{v}, z_{v}\right\}$ will depend on the number of buoys $N$.

If UAV uses a barometric depth gauge for determining $z_{v}$, the system of equations (1) takes the form

$$
\sqrt{\left(x_{i}-x_{v}\right)^{2}+\left(y_{i}-y_{v}\right)^{2}} \approx c T_{i}, \quad i=\overline{1, N}
$$

In this case, the solution of (14) is carried out as

$$
\left\{x_{v}, y_{v}\right\}=\arg \min _{x_{v}, y_{v}}\left[\sum_{i=1}^{N}\left(\sqrt{\left(x_{i}-x_{v}\right)^{2}+\left(y_{i}-y_{v}\right)^{2}}-c T_{i}\right)\right]
$$

Solving the system of equations (16 allows us to calculate the spoofer's coordinates $\left\{x_{s}, y_{s}\right\}$ as

$$
\left\{x_{s}, y_{s}\right\}=\arg \min _{x_{s}, y_{s}}\left[\sum_{i=1}^{N}\left(\sqrt{\left(x_{i}-x_{s}\right)^{2}+\left(y_{i}-y_{s}\right)^{2}}-c T_{i}\right)\right]
$$


To determine the coordinates $\left\{x_{s}, y_{s}\right\}$ there is enough data from three buoys or three GNSS satellites if the spoofer is on the surface of the sea.

Suppose we know the target's coordinates $\left\{x_{v}, y_{v}, z_{v}\right\}$, for example, using a sonar range finder and a measured direction to the target. If UAVdoes not use barometric depth gauge for determining $z_{v}$, then in this case it is possible to determine the corrections $\Delta T_{i}$ for measured time $T_{i}$ so that the receiver of UAVwould calculate the fake coordinates equal to the true ones (17).

$$
\left\{x_{v}, y_{v}, z_{v}\right\}=\arg \min _{x_{v}, y_{v}, z_{v}}\left\{\sum_{i=1}^{N}\left(\sqrt{\left(x_{i}-x_{v}\right)^{2}+\left(y_{i}-y_{v}\right)^{2}+\left(z_{i}-z_{v}\right)^{2}}-\left(c T_{i}+\Delta T_{i}\right)\right\}\right.
$$

If the power of the spoofer's signal exceeds the power of buoy signals, the target's receiver switches to the false signal. Further, the spoofer applies an escaping spoofing strategy in accordance with the equation system

$$
\sqrt{\left[x_{i}-\left(x_{v}+\Delta x_{v}\right)\right]^{2}+\left[y_{i}-\left(y_{v}+\Delta y_{v}\right)\right]^{2}+\left(z_{v}+\Delta z_{v}\right)^{2}} \approx c T_{i}+\Delta T_{i}, \quad i=\overline{1, N}
$$

where $\left\{\Delta x_{v}, \Delta y_{v}, \Delta z_{v}\right\}$ - correction of target's coordinates, taking away UAV from its route. In this situation, the active spoofer is surface-bound so the values $\mathrm{Z}_{\mathrm{i}}=0$, i.e. they correspond to zero sea level.

The algorithm for finding $\Delta T_{i}, i=\overline{1, N}$ with given vectors $\left\{x_{v}, y_{v}, z_{v}\right\}$ and $\left\{\Delta x_{v}, \Delta y_{v}, \Delta z_{v}\right\}$ is not considered in this article.

\section{The Spoofing Detection using a single Hydrophone}

In the next two sections we will discuss the two methods of spoofing detection:

1) the method of measuring the coordinates of a moving UAV at two points on the route using a single hydrophone (in this case we use a conventional hydrophone, that is, the problem of practical implementation of spoofing detection for GNSS-like UPS is reduced only to programming);

2) the method of measuring the coordinates of UAV at two points of space using dual hydrophones.

We install a fixed single hydrophone on the spoofing detector. Note that may be in motion.

\section{a. The measurement of a distance between two positions of a single hydrophone in navigation mode}

The spoofing detector measures the coordinates of the hydrophone $H$, based on a real signal from buoys:

$$
\left\{\text { 资, } y_{v^{\prime}}, \hat{z}_{v^{\prime}}\right\}=\arg \min _{x_{v^{\prime}}, y_{v^{\prime}}, z_{v^{\prime}}}\left\{\sum_{i=1}^{N}\left(\sqrt{\left(x_{i}-x_{v^{\prime}}\right)^{2}+\left(y_{i}-y_{v^{\prime}}\right)^{2}+\left(z_{i}-z_{v^{\prime}}\right)^{2}}-c T_{i}\right)\right\}
$$

where $\left(x_{v^{\prime}}, y_{v^{\prime}}, z_{v^{\prime}}\right)$ - unknown exact coordinates of the hydrophone $H$ at the time $t^{\prime},\left(\hat{x}_{v^{\prime}}, \hat{y}_{v^{\prime}}, \hat{z}_{v^{\prime}}\right)$ - calculated coordinates of the hydrophone $H$ at the time $t^{\prime}$.

The spoofing detector again measures the XYZ of the hydrophone $H$ at the time $t^{\prime \prime}$ :

$$
\left\{\text { 资, } y_{v^{\prime \prime}}, \hat{z}_{v^{\prime \prime}}\right\}=\arg \min _{x_{v^{\prime \prime}}, y_{v^{\prime \prime}}, z_{v^{\prime \prime}}}\left\{\sum_{i=1}^{N}\left(\sqrt{\left(x_{i}-x_{v^{\prime \prime}}\right)^{2}+\left(y_{i}-y_{v^{\prime \prime}}\right)^{2}+\left(z_{i}-z_{v^{\prime \prime}}\right)^{2}}-c T_{i}\right)\right\}
$$

where $\left(x_{v^{\prime \prime}}, y_{v^{\prime \prime}}, z_{v^{\prime \prime}}\right)-$ unknown exact coordinates of the hydrophone $H$ at the time $t^{\prime \prime},\left(\hat{x}_{v^{\prime \prime}}, \hat{y}_{v^{\prime \prime}}, \hat{z}_{v^{\prime \prime}}\right)-$ calculated coordinates of the hydrophone $H$ at the time $t^{\prime \prime}$.

The measured distance between the hydrophone at the times $t^{\prime}$ and $t^{\prime \prime}$ 


$$
\hat{D}_{1-2}=\sqrt{\left(\frac{2}{1}-x_{v^{\prime \prime}}\right)^{2}+\left(\text { 资 }-y_{v^{\prime \prime}}\right)^{2}+\left(\text { 缷 }-z_{v^{\prime \prime}}\right)^{2}}
$$

must correspond with the distance traveled by the vehicle over time $\left(t^{\prime \prime}-t\right)$, i.e.

$$
\hat{D}_{1-2} \approx V\left(t^{\prime \prime}-t^{\prime}\right)
$$

\section{b. The measurement of a distance between the two positions of a single hydrophone in spoofing mode}

The spoofing detector measures the coordinates of the hydrophones $\mathbf{H}$, based on false signal from spoofer:

$$
\left\{\text { 资, } y_{v^{\prime}}, \hat{z}_{v^{\prime}}\right\}=\arg \min _{x_{v^{\prime}}, y_{v^{\prime}}, z_{v^{\prime}}}\left\{\sum_{i=1}^{N}\left(\sqrt{\left(x_{i}-x_{v^{\prime}}\right)^{2}+\left(y_{i}-y_{v^{\prime}}\right)^{2}+\left(z_{i}-z_{v^{\prime}}\right)^{2}}-c T_{i}\right)\right\}
$$

where $\left(x_{v^{\prime}}, y_{v^{\prime}}, z_{v^{\prime}}\right)$ - unknown precise coordinates of the hydrophone $H$ at the time $t^{\prime},\left(\hat{x}_{v^{\prime}}, \hat{y}_{v^{\prime}}, \hat{z}_{v^{\prime}}\right)$ - calculated coordinates of the hydrophone $H$ at the time $t^{\prime}$.

The spoofing detector again measures the XYZ of the hydrophone $H$ at the time $t^{\prime \prime}$

$$
\left\{\text { 资, } y_{v^{\prime \prime}}, \hat{z}_{v^{\prime \prime}}\right\}=\arg \min _{x_{v^{\prime}}, y_{v^{\prime}}, z_{v^{\prime \prime}}}\left\{\sum_{i=1}^{N}\left(\sqrt{\left(x_{i}-x_{v^{\prime \prime}}\right)^{2}+\left(y_{i}-y_{v^{\prime \prime}}\right)^{2}+\left(z_{i}-z_{v^{\prime \prime}}\right)^{2}}-c T_{i}\right)\right\}
$$

where $\left(x_{v^{\prime \prime}}, y_{v^{\prime \prime}}, z_{v^{\prime \prime}}\right)$ - unknown exact coordinates of the hydrophone $H$ at the time $t^{\prime \prime},\left(\hat{x}_{v^{\prime \prime}}, \hat{y}_{v^{\prime \prime}}, \hat{z}_{v^{\prime \prime}}\right)-$ calculated coordinates of the hydrophone $H$ at the time $t^{\prime \prime}$.

The measured distance between the hydrophone $H$ at the time $t^{\prime}$ and the hydrophone $\mathrm{Y}$ at the time $t^{\prime \prime}$

$$
\hat{D}_{1-2}=\sqrt{\left(\text { 资 }-x_{v^{\prime \prime}}\right)^{2}+\left(\text { 瓷 }-y_{v^{\prime \prime}}\right)^{2}+\left(\text { 資 }-z_{v^{\prime \prime}}\right)^{2}} \approx 0
$$

because all hydrophones in the spoofing zone calculate the same false coordinates and $\hat{D}_{1-2}$ must be not corresponding with the distance traveled by the vehicle over time $\left(t^{\prime \prime}-t^{\prime}\right)$, i.e.

$$
\hat{D}_{1-2}<<\left(t^{\prime \prime}-t^{\prime}\right)
$$

\section{c. The decisive rule}

Comparing (24) and (25), we can write down the decisive rule for the detection of spoofing

$$
\text { if } \hat{D}_{1-2} \leq \breve{D} \text { then go to Spoofing, }
$$

where $\breve{D}$ - discriminant determined on the basis of statistical studies at the stage of designing a real detection system. At present, we are carrying out theoretical studies and relevant real sea tests at various speeds $V$ and various values $\Delta t=\left(t^{\prime \prime}-t^{\prime}\right)$ in order to find acceptable values of $\breve{D}$.

Note that the spoofing detector may be in motion. During the time $\Delta t=\left(t^{\prime \prime}-t^{\prime}\right)$, the parameters of the spoofer's signals may change, therefore when solving the problem of optimizing the parameters of the spoofing detector it then becomes necessary to minimize the parameter $\Delta t$. From the point of view of the detection of spoofing, it is necessary to maximize the parameter $\Delta t$. To resolve this contradiction, minimax methods of parametric optimization are used [16]. Minimax is a type of backtracking algorithm that is used in decisionmaking and game theory to find the optimal move for a player, assuming that your opponent also plays optimally. It is widely used in two player turn-based games such as Tic-Tac-Toe, Backgammon, Mancala, Chess, etc. 


\section{Spoofing Detection using a Dual Hydrophone}

We install two fixed hydrophones $H^{\prime}$ and $H^{\prime \prime}$ on the spoofing detector at distance $D$ from each other. Note that the spoofing detector may be in immobile or in motion.

\section{A. The measurement of the distance between hydrophones in navigation mode}

The spoofing detector measures the coordinates of the hydrophone $H^{\prime}$ :

$$
\left\{\text { 资, } y_{v^{\prime}}, \hat{z}_{v^{\prime}}\right\}=\arg \min _{x_{v^{\prime}}, y_{v^{\prime}}, z_{v^{\prime}}}\left\{\sum_{i=1}^{N}\left(\sqrt{\left(x_{i}-x_{v^{\prime}}\right)^{2}+\left(y_{i}-y_{v^{\prime}}\right)^{2}+\left(z_{i}-z_{v^{\prime}}\right)^{2}}-c T_{i}\right)\right\}
$$

where $\left(x_{v^{\prime}}, y_{v^{\prime}}, z_{v^{\prime}}\right)$ - unknown exact coordinates of the hydrophone $H^{\prime},\left(\hat{x}_{v^{\prime}}, \hat{y}_{v^{\prime}}, \hat{z}_{v^{\prime}}\right)$ - calculated coordinates of the hydrophone $H^{\prime}$.

The spoofing detector measures the coordinates of the hydrophone $H^{\prime \prime}$ :

$$
\left\{\text { 资, } y_{v^{\prime \prime}}, \hat{z}_{v^{\prime \prime}}\right\}=\arg \min _{x_{v^{\prime \prime}}, y_{v^{\prime \prime}}, z_{v^{\prime \prime}}}\left\{\sum_{i=1}^{N}\left(\sqrt{\left(x_{i}-x_{v^{\prime \prime}}\right)^{2}+\left(y_{i}-y_{v^{\prime \prime}}\right)^{2}+\left(z_{i}-z_{v^{\prime \prime}}\right)^{2}}-c T_{i}\right)\right\}
$$

where $\left(x_{v^{\prime \prime}}, y_{v^{\prime \prime}}, z_{v^{\prime \prime}}\right)-$ unknown exact coordinates of the hydrophone $H^{\prime \prime}$ at the time $t^{\prime},\left(\hat{x}_{v^{\prime \prime}}, \hat{y}_{v^{\prime \prime}}, \hat{z}_{v^{\prime \prime}}\right)-$ calculated coordinates of the hydrophone $H^{\prime \prime}$.

The measured distance between $H^{\prime}$ and $H^{\prime \prime}$ is

$$
\hat{D}_{1-2}=\sqrt{\left(\hat{x}_{v^{\prime}}-\hat{x}_{v^{\prime \prime}}\right)^{2}+\left(\hat{y}_{v^{\prime}}-\hat{y}_{v^{\prime \prime}}\right)^{2}+\left(\hat{z}_{v^{\prime}}-\hat{z}_{v^{\prime \prime}}\right)^{2}} \approx D
$$

where $D$ - the real distance between hydrophones.

B. The measurement of the distance between hydrophones in spoofing mode

Because all hydrophones in the spoofing zone calculate the same false coordinates the equation (29) takes the form

$$
\hat{D}_{1-2}=\sqrt{\left(\text { 资 }-x_{v^{\prime \prime}}\right)^{2}+\left(\text { 畩 }-y_{v^{\prime \prime}}\right)^{2}+\left(\text { 疼 }-z_{v^{\prime \prime}}\right)^{2}} \approx 0 \text {. }
$$

C. The decisive rule

Comparing (29) and (30), we can write down the decisive rule for the detection of spoofing

$$
\text { if } \hat{D}_{1-2} \leq \breve{D} \text { then go to Spoofing, }
$$

where $\breve{D}$-a discriminant determined on the basis of statistical studies at the stage of designing a real detection system.

\section{Discussion}

In the process of underwater positioning systems designing, we have repeatedly response to the following questions.

\section{Q1. Is it possible to build an underwater GNSS?}

A1. No. It is currently possible to build LNSS - Local Navigation Satellite System.

Q2. What is the size of LNSS?

A2. The size of LNSS can reach several square kilometers.

Q3. What is the depth of LNSS?

A3. The depth of LNSS can reach a few hundred meters.

Q4. What are the main steps of GNSS vessel spoofing? 
A4. GNSS receiver of vessel calculates the true location based on real GNSS satellites;

GNSS receiver sends accurate location information to Automatic Identification System (AIS);

GNSS spoofing transmitter mimics real GNSS satellites and broadcasts false navigation signals;

GNSS receiver calculates false location based on signals from GNSS spoofing transmitter;

GNSS receiver sends false location information to AIS;

Divert vessel into hostile or territorial waters.

\section{Q5. What are the main steps of UAV spoofing?}

A5. LNSS receiver calculates the true location based on real GIB (GPS intelligent buoys) [9];

LNSS spoofing transmitter mimics real GIB and broadcasts false acoustic navigation signals;

LNSS receiver calculates false location based on signals from LNSS spoofing transmitter;

Divert underwater transport into hostile or territorial waters.

\section{Q6. Can you give examples of LNSS spoofing?}

A6. We do not know examples of underwater spoofing. Underwater positioning systems are in the early stages, but the development of underwater robotics will require protection from possible terrorist attacks.

\section{Q7. What is better to use a system with one hydrophone or with two hydrophones?}

A7. Spoofing detection systems with one hydrophone are of great practical importance, as in this case it is possible to use standard hydro acoustic equipment

\section{Conclusions}

The results of our studies have shown that the formal transfer direct transference of GNSS under water is impossible, since the underwater positioning systems are local in nature and the physics of acoustic waves in water and electromagnetic waves in the atmosphere are fundamentally different. However, the basic techniques, which are used to solve the problem of spoofing detection above water, can also be used under water. Since conduction of physical experiments underwater is incomparably more complex task than surface experiments, at this stage of research we tested the principles of underwater spoofing detection using a simulation approach.

\section{References}

[1] C4ADS Innovation for peace. https://safety4sea.com/wp-content/uploads/2019/04/C4ADSAbove-us-only-start_Exposing-GPS-spoofing-in-Russia-and-Syria-2019_04.pdf [Accessed:

October 20, 2019]

[2] EvoLogics, Underwater Acoustic LBL Positioning Systems, 2018 // https://evologics.de/underwater-positioning [Accessed: October 20, 2019]

[3] Sonardyne, Subsea technology for energy, science and security // https://www.sonardyne.com [Accessed: October 20, 2019]

[4] BAE Systems (2016) Undersea navigation and positioning system development to begin for U.S. Navy. // https://www.baesystems.com/en-us/what-we-do/cyber-security---intelligence [Accessed: October 20, 2019]

[5] N. Lavars, DARPA program plunges into underwater positioning system. [Online] 23 May 2016. Available from: https://newatlas.com/darpa-underwater-navigation/43472/ [Accessed: October 20, 2019]

[6] J. Waterston, Positioning System for Deep Ocean Navigation (POSYDON) https://www.darpa.mil/program/positioning-system-for-deep-ocean-navigation [Accessed: October 20, 2019]

[7] K. Osborn, DARPA Discovers "GPS-Like" undersea drone connectivity, Feb 14, 2017 // https://defensesystems.com/articles/2017/02/14/darpauuv.aspx [Accessed: October 20, 2019] 
[8] Scuba Diving Chicago, Underwater Vehicles, 18 Apr 2013 Underwater GPS navigation // https://www.scubadivingchicago.us/underwater-vehicles/underwater-gps-navigation.html [Accessed: October 20, 2019]

[9] H.G. Thomas, GIB buoys: an interface between space and depths of the oceans. Proceedings of the 1998 Workshop on Autonomous Underwater Vehicles, 21 Aug. 1998, pp. 181-184.

Available from: https://ieeexplore.ieee.org/abstract/document/744453 [Accessed: October 20, 2019]

[10] JANUS creates a new era for digital underwater communications. https://robohub.org/januscreates-a-new-era-for-digital-underwater-communications/ [Accessed: October 20, 2019]

[11] Hubert, T. Method and device for the monitoring and remote control of unmanned, mobile underwater vehicles. United States Patent 5,579.285, (1966) https://patents.google.com/patent/US5579285A/en [Accessed: October 20, 2019]

[12] J.W. Youngberg, A Novel Method for Extending GPS to Underwater Applications. Navigation 38,1991 , pp. 263-271.

[13] M. Caparrini, A. Egido, F. Soulat, O. Germain, E. Farres, S. Dunne \& G. Ruffini, Oceanpal®: monitoring sea state with a GNSS-R coastal instrument. Paper presented at the International Geoscience and Remote Sensing Symposium. IEEE, Barcelona, Spain, 23-28 July 2007, doi:10.1109/IGARSS.2007.4424004

[14] P. Zalewski, Real-time GNSS spoofing detection in maritime code receivers. Scientific Journals of Maritime University of Szczecin2014, 38(110) pp. 118-124 http://repository.scientificjournals.eu/handle/123456789/608 [Accessed: October 20, 2019]

[15] E.Ochin, Ł.Lemieszewski, E.Lusznikov, L.Dobryakova, The study of the spoofer's some properties with help of GNSS signal repeater. Scientific Journals of the Maritime University of Szczecin 36 (108) 2013 z.2, pp. 159-165 http://repository.scientificjournals.eu/handle/123456789/581 [Accessed: October 20, 2019]

[16] M. Ehrgott, J. Ide \& A. Schöbel, Minmax robustness for multi-objective optimization. European Journal of Operational Research 239, 1, 2014, pp. 17-31. 